\title{
On the Weak Adequacy of Branching-Time Temporal Logic
}

\author{
Ph. Schnoebelen and S. Pinchinat* \\ Laboratoire d'Informatique Fondamentale \\ et d'Intelligence Artificielle, \\ Institut Imag - CNRS, \\ Grenoble - FRANCE
}

\begin{abstract}
.
We study the adequacy of branching-time temporal logic w.r.t. bisimulation semantics in the framework of non-deterministic programs without the finitely-branching restriction. The process equivalence generated by branching-time logic is compared with bisimulation and with two observational equivalences. It is found at best weakly adequate. This further illustrates the strength of the finitely-branching restriction. However, we argue that in connection with branching time temporal logic, one has no better choice than bisimulation as a semantic equivalence: in particular, the equivalence generated by temporal logic is not a congruence w.r.t. usual process operators.
\end{abstract}

\section{Introduction}

When using temporal logic to reason about parallel or non-deterministic programs, a general prerequisite is to investigate the adequacy of the logic with the semantics one considers for its (parallel) programs. Basically, the logic should not be able to distinguish two programs that we want to consider as semantically equivalent, or more formally:

$$
P \simeq P^{\prime} \Rightarrow \text { for all } f\left(P \models f \text { iff } P^{\prime} \models f\right)
$$

using $\simeq$ to denote semantic equivalence and writing $P \models f$ when program $P$ satisfies formula $f$. Let us write $T h(P)$ for $\{f \mid P \models f\}$, so that the previous requirement is written:

$$
P \simeq P^{\prime} \Rightarrow T h(P)=T h\left(P^{\prime}\right)
$$

If this requirement is not met, one cannot meaningfully consider the logic as "speaking about" semantic objects. A stronger requirement is

$$
P \simeq P^{\prime} \Leftrightarrow T h(P)=T h\left(P^{\prime}\right)
$$

which holds if the logic is sufficiently powerful to distinguish any two semantically different programs (and only these). We say that the logic is adequate when (2) holds, and weakly

*LIFLA-IMA, 46 Av. Felix Viallet, 38031 GRENOBLE Cedex, FRANCE. E-mait:\{phs,sp\}olifis.imag.fr 
adequate when we only have (1).

Lamport and Pnueli initiated with [Pnu77,Lam80] the use of temporal logic for reasoning about parallel or non-deterministic programs. [Lam80] also initiated the controversy about branching-time vs. linear-time temporal logic. This debate mainly focused on the different expressiveness of the proposed logics and culminated with the introduction of $C T L^{*}$ [EH83], a logic including both branching-time and linear-time constructs, and subsuming several other proposals.

The adequacy of a temporal logic was first investigated by Hennessy and Milner [HM85] who studied bisimulation (from [Par81]) as a semantic equivalence for processes (parallel programs). They proved that bisimulation was exactly characterized by a very simple modal language, the so-called Hennessy-Milner Logic: two processes are bisimilar iff they cannot be distinguished by HML temporal formulas. This was a strong point in favor of bisimulation as a semantic equivalence (see also [Abr87,BK89]).

The adequacy result of [HM85] is true of many branching-time temporal logics. However, it only applies to finitely-branching systems (i.e. systems where in any given state, there is only a finite number of possible choices), hereafter abbreviated as f.b. systems. In the general case of not necessarily f.b. systems, the proof of [HM85] only yields weak adequacy. However, several parallel languages do not want to restrict themselves to f.b. systems e.g. because these are not closed under some natural operations (communication of values, ...) [BK89,GR83]. We have that same problem with our own FP2 language [SJ89], which led us to investigate the adequacy of the $C T L$ temporal logic (from [CE81]) w.r.t. bisimulation semantics in non-f.b. systems. The technical literature rarely considers non-f.b. systems: we only found [BR83] as directly addressing our problem. Other works (e.g. [HS85]) only mention adequacy results for logics that admit infinite (not necessarily countable !) conjunctions.

In this paper, we show that branching-time temporal logic is only weakly adequate w.r.t. bisimulation (written $\leftrightarrow$ ):

$$
P_{\leftrightarrows} P^{\prime} \stackrel{\notin}{\Rightarrow} T h(P)=T h\left(P^{\prime}\right)
$$

Trying to find a different semantics w.r.t. which temporal logic could be adequate, we investigate Milner's original strong observational equivalence (written $\equiv$ ). We show that it is too broad:

$$
P \equiv P^{\prime} \underset{\not}{\nRightarrow} T h(P)=T h\left(P^{\prime}\right)
$$

There remains one natural intermediate semantic equivalence, adapted from $\equiv$ and written $\approx$, that fits between $\leftrightarrows$ and $\equiv$. We show:

$$
P \approx P^{\prime} \nRightarrow T h(P)=T h\left(P^{\prime}\right)
$$

Seeing that $C T L$ equivalence fails to match any of three natural semantic equivalences, a question one may ask is "why not consider it as our semantic equivalence ?". We investigated this question in [Sch89] where we show that $C T L$ equivalence does not yield a congruence 
w.r.t. parallel composition (but it is a congruence w.r.t. several other process operations) so that we end up arguing that if one wants to use branching-time temporal logic without the f.b. restriction, one should stick with bisimulation semantics and be content with weak adequacy. Note also that with the f.b. restriction, everything collapses into:

$$
P \Leftrightarrow P^{\prime} \Leftrightarrow P \equiv P^{\prime} \Leftrightarrow P \approx P^{\prime} \Leftrightarrow T h(P)=T h\left(P^{\prime}\right)
$$

In this paper we used $C T L$ for its conceptual simplicity and widespread use but the aforementioned results are general and, modulo some minor details, they apply equally well to other (branching-time) logics (we deal with $C T L^{*}$ in Appendix A). By contrast, [BR83] only considered logics unable to express eventual termination. As a consequence, (5) was specialized into

$$
P \approx P^{\prime} \nRightarrow T h(P)=T h\left(P^{\prime}\right)
$$

As another point of comparison with [BR83], we provide simpler counter-examples based on the ordinal processes of Klop.

In section 1, we recall the basic notions and results we shall use: graphs, bisimulation, the $C T L$ temporal logic and its semantics. Then, the following sections compare $C T L$ with all three equivalences, in turn. The main innovations of the paper are Propositions 3 and 4, relying on the counter-examples developed for Lemmas 2 and 3 . The picture is completed with Propositions 1 and 2. Finally, an appendix contains results that are mentioned in the text, but that are out of the precise scope of the paper.

\section{Preliminaries}

Definition 1 (Graph) $A$ (directed) graph is a pair $P=(Q, \rightarrow)$ where $Q$ is any set of states, with typical element $q$, and $\rightarrow \subseteq Q \times Q$ is any transition relation.

For any $k \in \mathrm{N}$, we write $\stackrel{k}{\rightarrow}$ for the $k$-fold composition of $\rightarrow: \stackrel{k}{\rightarrow}=\rightarrow 0 \cdots \circ \rightarrow(k$-times) and $\stackrel{0}{\rightarrow}=I d_{Q}$. We write $q \rightarrow q^{\prime}$ when $\left(q, q^{\prime}\right) \in \rightarrow$ and say that $q^{\prime}$ is a successor of $q$. A terminal state is a state with no successors.

A path is a maximal sequence $q_{0} \rightarrow q_{1} \rightarrow q_{2} \rightarrow \cdots$ of states of $Q$, it may be finite (and ending with a terminal state) or infinite. Given a path $\sigma=q_{0} \rightarrow q_{1} \rightarrow q_{2} \rightarrow \cdots$, we write $|\sigma|$ for the length (number of steps) of $\sigma$ : it may be infinite, it may also be 0 if $q_{0}$ is terminal. When $|\sigma| \geq i$, we write $\sigma(i)$ for the state $q_{i}$ and $\sigma^{i}$ for the suffix path $q_{i} \rightarrow q_{i+1} \rightarrow \ldots$ We write $\Sigma(q)=\{\sigma, \pi, \ldots\}$ for the set of all paths starting from $q$ and let $\Sigma$ denote $\bigcup_{q \in Q} \Sigma(q)$.

In the following, only states from a same graph will be compared. This is no loss of generality as any state can be seen as the rooted directed connected sub-graph it canonically induces. Dually, comparing two (rooted) graphs must be understood as comparing their roots in some union graph. In this paper graph nodes, rooted graphs and processes are the same concepts.

We say that $\rightarrow$ is finitely branching (or image-finite, or f.b.) if for any $q \in Q$, the set $\left\{q^{\prime} \in Q \mid q \rightarrow q^{\prime}\right\}$ is finite. We explicitly do not restrict ourselves to f.b. graphs. 


\subsection{Three process equivalences}

Given $P=(Q, \rightarrow)$, we define three equivalence relations over $Q$ :

Definition 2 (Bisimulation) $\leftrightarrows \subseteq Q \times Q$ is the largest relation over $Q$ s.t. $q_{1} \leftrightarrow q_{2}$ iff:

- $\forall q_{1}^{\prime} \in Q$, if $q_{1} \rightarrow q_{1}^{\prime}$ then $\exists q_{2}^{\prime} \in Q$ s.t. $q_{2} \rightarrow q_{2}^{\prime}$ and $q_{1}^{\prime} \uplus q_{2}^{\prime}$,

- and reciprocally, $\forall q_{2}^{\prime} \in Q$, if $q_{2} \rightarrow q_{2}^{\prime}$ then $\exists q_{1}^{\prime} \in Q$ s.t. $q_{1} \rightarrow q_{1}^{\prime}$ and $q_{1}^{\prime} \leftrightarrows q_{2}^{\prime}$.

Definition 3 (Projective equivalences) We define $q_{1} \equiv q_{2}$ iff $q_{1} \equiv_{n} q_{2}$ for all $n \in \mathrm{N}$, where $\equiv_{n}$ is given inductively by:

- $q_{1} \equiv_{0} q_{2}$ always,

- $q_{1} \equiv_{n+1} q_{2}$ iff

$-\forall q_{1}^{\prime} \in Q$, if $q_{1} \rightarrow q_{1}^{\prime}$ then $\exists q_{2}^{\prime} \in Q$ s.t. $q_{2} \rightarrow q_{2}^{\prime}$ and $q_{1}^{\prime} \equiv_{n} q_{2}^{\prime}$,

- and reciprocally.

Similarly, we define $q_{1} \approx q_{2}$ iff $q_{1} \approx_{n} q_{2}$ for all $n \in \mathbf{N}$, where $\approx_{n}$ is given inductively by:

- $q_{1} \approx_{0} q_{2}$ always,

- $q_{1} \approx_{n+1} q_{2}$ iff

$-\forall k \in \mathrm{N}, \forall q_{1}^{\prime} \in Q$, if $q_{1} \stackrel{k}{\rightarrow} q_{1}^{\prime}$ then $\exists q_{2}^{\prime} \in Q$ s.t. $q_{2} \stackrel{k}{\rightarrow} q_{2}^{\prime}$ and $q_{1}^{\prime} \approx_{n} q_{2}^{\prime}$,

- and reciprocally.

All three relations are clearly equivalence relations. The name "projective equivalence" comes from [BK87] where, writing $(x)_{n}$ for the truncation of $x$ at $\operatorname{depth} n, x \equiv_{n} y$ is defined as $(x)_{n} \leftrightarrow(y)_{n}$.

Another reading of the previous definitions involves a mapping $\mathcal{F}$ over the complete lattice $2^{Q \times Q}$ of relations over $Q$ [Mil88]. For $R \subseteq Q \times Q, \mathcal{F}(R)$ is defined by $q_{1} \mathcal{F}(R) q_{2}$ iff $q_{1} \rightarrow q_{1}^{\prime}$ implies that there exists $q_{2} \rightarrow q_{2}^{\prime}$ s.t. $q_{1}^{\prime} R q_{2}^{\prime}$, and reciprocally. $\mathcal{F}$ is monotonic and satisfies $I d_{Q} \subseteq$ $\mathcal{F}\left(I d_{Q}\right)$. Clearly $\equiv$ is defined as $\bigcap_{n \in \mathbf{N}} \mathcal{F}^{n}(Q \times Q)$. Similarly, $\approx$ is defined as $\bigcap_{n \in \mathbf{N}} \mathcal{G}^{n}(Q \times Q)$ for some monotonic $\mathcal{G}$. As $\mathcal{G}(R) \subseteq \mathcal{F}(R)$ for any $R \subseteq Q \times Q$, we get $\approx \subseteq \equiv$. Furthermore, $\leftrightarrow$ is defined as the largest fixpoint of $\mathcal{F}$ (or, equivalently, of $\mathcal{G}$ ). Monotonicity of $\mathcal{F}$ (and of $\mathcal{G}$ ) entails that $\leftrightarrows$ is correctly defined (it is $\bigcup\{R \mid R \subseteq \mathcal{F}(R)\}$ ) and that $\leftrightarrow \subseteq \equiv$ (and $\leftrightarrow \subseteq \approx$ ). It is well-known that if $\rightarrow$ is f.b., $\mathcal{F}$ is anticontinuous, which implies that $\bigcap_{n \in \mathrm{N}} \mathcal{F}^{n}(Q \times Q)$ is the largest fixpoint of $\mathcal{F}$, i.e. $\equiv=\approx=\leftrightarrows$ for f.b. graphs. However, in general, the inclusions are strict (this is already known, but our comparison with $C T L$ will yield another proof).

We extend the notion of bisimulation to paths: $\sigma \leftrightarrow \pi$ iff $\sigma(i) \leftrightarrows \pi(i)$ for every $i$. Then, maximality of paths implies that $\sigma$ and $\pi$ have same length. Also, this implies that $\sigma^{i} \leftrightarrows \pi^{i}$ for any $i$. Note that if $q \leftrightarrow q^{\prime}$ and if $\sigma \in \Sigma(q)$, we can build $\sigma^{\prime} \in \Sigma\left(q^{\prime}\right)$ s.t. $\sigma \leftrightarrow \sigma^{\prime}$. 


\subsection{Ordinal processes}

In several cases, we shall use the ordinal processes of [Klo88], or a variant of them. We recall here their definition and main properties:

Definition 4 (Ordinal processes) Given any ordinal $\lambda$, the ordinal process $P_{\lambda}$ is the graph $(\lambda,>)$.

Thus, in $P_{\lambda}$ we have $\alpha \rightarrow \beta$ iff $\beta<\alpha<\lambda$. A basic property is that $P_{\lambda}$ has no infinite paths and that $\rightarrow$ is transitive. $P_{\lambda}$ is non-f.b, as soon as $\lambda>\omega$. Clearly, over $P_{\lambda}, \leftrightarrow$ is reduced to equality. Indeed, suppose $\alpha_{0} \uplus \alpha_{1}$ with $\alpha_{1}<\alpha_{0}<\lambda$. Then $\alpha_{0} \rightarrow \alpha_{1}$, and $\alpha_{0} \leftrightarrow \alpha_{1}$ implies that there exists $\alpha_{1} \rightarrow \alpha_{2}$ s.t. $\alpha_{1} \leftrightarrow \alpha_{2}$. Carrying on, we shall build an infinite path $\alpha_{0} \rightarrow \alpha_{1} \rightarrow \alpha_{2} \rightarrow \cdots$ contradicting the well-foundedness of $>$ over ordinals.

Conceptually, all states of an ordinal process are different (w.r.t. bisimilarity). Now, given an equivalence $\sim$ over $\lambda$, we say that $\sim$ distinguishes up to $\beta \leq \lambda$ iff, for any $\alpha_{1}, \alpha_{2}<\beta$, $\alpha_{1} \sim \alpha_{2}$ implies $\alpha_{1}=\alpha_{2}$. Clearly, $\equiv_{n}$ distinguishes up to $n$, so that $\equiv$ distinguishes up to $\omega$. Similarly, it is easy to prove (see Lemma 3 for a similar proof) that $\approx_{n}$ distinguishes up to $\omega . n$, so that $\approx$ distinguishes up to $\omega^{2}$. (In this terminology, $\leftrightarrow$ distinguishes up to any $\lambda$ ).

\subsection{The CTL temporal logic}

Definition 5 (Syntax of $C T L$ )

$$
(C T L \ni) f, g::=\top|f \wedge g| \neg f|\mathbf{E X} f| \mathbf{E} f \mathbf{U} g \mid \mathbf{A} f \mathbf{U} g
$$

Other logical connectives are definable in terms of $T, \wedge, \neg$ and EX : $\perp$ is $\neg T, f \vee g$ is $\neg(\neg f \wedge \neg g)$, and $\mathbf{A X} f$ is $\neg \mathbf{E X} \neg f$.

$C T L$ formulas denote properties of states in a graph: given a graph $P=(Q, \rightarrow)$, a state $q \in Q$ and some formula $f \in C T L$, we write $q \vDash_{P} f$ to denote that, in graph $P, f$ holds in $q$ or that $q$ satisfies $f$. Similarly $q \not \neq_{P} f$ means that $f$ does not hold in $q$. We always drop the $P$ subscript when it is clear from the context.

We define $\models \subseteq Q \times C T L$ in the standard way, by induction over the structure of formulas:

Definition 6 (Semantics of $C T L$ ) For all $q, q_{0} \in Q$ and $f, g \in C T L$ :

- $q \models T, q \models f \wedge g$ and $q \models \neg f$ have their obvious definitions.

- $q \models \mathbf{E X} f$ iff there exists $q \rightarrow q^{\prime}$ s.t. $q^{\prime} \vDash f$.

- $q_{0} \vDash \mathbf{E} f \mathbf{U} g$ iff there exists a path $q_{0} \rightarrow q_{1} \rightarrow q_{2} \rightarrow \cdots$ and some $n \in \mathbf{N}$ s.t. $q_{n} \vDash g$ and $q_{i} \vDash f$ for all $i<n$.

- $q_{0} \vDash \mathbf{A} f \mathbf{U} g$ iff for all paths $q_{0} \rightarrow q_{1} \rightarrow q_{2} \rightarrow \cdots \in \Sigma\left(q_{0}\right)$ there is some $n \in \mathbf{N}$ s.t. $q_{n} \vDash g$ and $q_{i} \neq f$ for all $i<n$.

$C T L$ is paradigmatic in the field of branching-time temporal logic because it admits efficient model checking algorithms while remaining very expressive (see [ES89] for a survey). EX $f$ can 
be read as "there exists a next state satisfying $f$ ", $\mathbf{E} f \mathbf{U} g$ as "there exists a path (starting from here) satisfying $f$ all along until $g$ is eventually satisfied", and $\mathbf{A} f \mathbf{U} g$ as "all paths (starting from here) satisfy $f$ all along until $g$ is eventually satisfied". by:

Given $(Q, \rightarrow)$ and $q \in Q$, we write $T h(q)$ for $\{f \in C T L \mid q \vDash f\}$ and define $\stackrel{\text { CTL }}{\sim} \subseteq Q \times Q$

$$
q^{C T L} q^{\prime} \text { iff } T h(q)=T h\left(q^{\prime}\right)
$$

(that is, iff $q$ and $q^{\prime}$ are elementarily equivalent in model-theoretic terminology). This is clearly an equivalence relation. A similar construction applies to any set of formulas, and we shall freely write $q_{\sim}^{F} q^{\prime}$ for any set $F \subseteq C T L$. Clearly, $F \subseteq C T L$ implies $\stackrel{c T L}{\sim} \subseteq \stackrel{F}{\sim}$.

\section{Comparing $C T L$ and $\leftrightarrows$}

Proposition 1 For any graph $(Q, \rightarrow)$, and states $q, q^{\prime} \in Q$ :

$$
q \leftrightarrow q^{\prime} \Rightarrow q^{c T L} q^{\prime}
$$

This is well-known. One proves that $q \leftrightarrow q^{\prime}$ implies $\left(q \models f\right.$ iff $\left.q^{\prime} \models f\right)$ by induction over the structure of $f$. As an example let us consider the case where $f$ is $\mathbf{A} g_{1} \mathbf{U} g_{2}$. Assume that $q \not \neq f$. Then there is a path $\sigma \in \Sigma(q)$ s.t. no $\sigma(i)$ satisfy $g_{2}$, or such that $\sigma(n) \not g_{1}$ for some $n$ with $\sigma(i) \notin g_{2}$ for all $i \leq n$. By bisimilarity, we can build a path $\sigma^{\prime} \in \Sigma\left(q^{\prime}\right)$ s.t. $\sigma \leftrightarrow \sigma^{\prime}$. Then for any $i, \sigma(i) \leftrightarrow \sigma^{\prime}(i)$, which implies (by ind. hyp.) that $\sigma^{\prime}(i) \models g_{1}$ iff $\sigma(i) \models g_{1}$ (and similarly for $\left.g_{2}\right)$. Finally, $\sigma^{\prime}$ is such that $q^{\prime} \not \neq f$. All other possible constructions for $f$ are dealt with in the same way.

Of course, the reverse implication does not hold, as a corollary of Proposition 3.

\section{Comparing $C T L$ and $\equiv$}

A concept we shall need now and later is the modal height (w.r.t. EX) of a formula. Formally, we define $d: C T L \rightarrow \mathrm{N}$ by:

$$
\begin{array}{rlrl}
d(T) & =0 & d(\mathbf{E X} f) & =d(f)+1 \\
d(f \wedge g) & =\max (d(f), d(g)) & d(\mathbf{A} f \mathbf{U} g) & =\max (d(f), d(g)) \\
d(\neg f) & =d(f) & d(\mathbf{E} f \mathbf{U} g) & =\max (d(f), d(g))
\end{array}
$$

We now consider the fragment $H M L$ of $C T L$, built using only $\top, \neg, \wedge, \mathbf{E X}$ :

$$
(H M L \ni) f, g::=T|f \wedge g| \neg f \mid \mathbf{E X} f
$$

and we stratify it according to the modal heights. More precisely, for any $n \in \mathbf{N}$, we define $H M L_{n}=\{f \in H M L \mid d(f) \leq n\}$. We have $H M L_{0} \subseteq H M L_{1} \subseteq \cdots \subseteq H M L_{n} \subseteq \cdots \subseteq$ $H M L \subseteq C T L$. Modulo our use of unlabeled steps, $H M L$ is the so-called Hennessy-Milner logic used in [HM85].

Note that, modulo the usual boolean laws, there exists only a finite number of distinct formulas in any $H M L_{n}$ set, so that any ${ }^{H M L_{n}} \sim$ equivalence generates only a finite number of equivalence classes. 
Lemma 1 For any graph $(Q, \rightarrow)$, and states $q_{1}, q_{2} \in Q$ :

$$
q_{1} \stackrel{H M L_{n}}{\sim} q_{2} \Leftrightarrow q_{1} \equiv_{n} q_{2}
$$

Proof We proceed by induction on $n$. The proof of [HM85] relies on the f.b. hypothesis, so that we have to invoke another argument for the " $\Rightarrow$ " direction.

$(\Rightarrow)$ : Clearly $\stackrel{B M L_{0}}{\sim} \subseteq \equiv_{0}$. Now assume that $\stackrel{B M L_{n}}{\sim} \subseteq \equiv_{n}$ and consider two states $q_{1}, q_{2}$ with $q_{1}{ }^{H M L_{n+1}} q_{2}$. If $q_{1} \rightarrow q_{1}^{\prime}$, we partition $H M L_{n}$, which is finite, into formulas satisfied at $q_{1}^{\prime}$ and formulas not satisfied at $q_{1}^{\prime}$, that is we write $H M L_{n}=\left\{f_{1}, \ldots, f_{m}\right\} \cup\left\{f_{m+1}, \ldots, f_{p}\right\}$ with $q_{1}^{\prime} \vDash f_{1} \wedge \cdots \wedge f_{m} \wedge \neg f_{m+1} \wedge \cdots \wedge \neg f_{p}$. Now, writing $f^{*}$ for $f_{1} \wedge \cdots \wedge f_{m} \wedge \neg f_{m+1} \wedge \cdots \wedge \neg f_{p}$, we have $d\left(f^{*}\right) \leq n$ so that (a boolean equivalent of) $f^{*}$ belongs to $H M L_{n}$ and $\mathbf{E X} f^{*} \in H M L_{n+1}$. $q_{1}^{\prime} \models f^{*}$ innplies $q_{1} \models \mathbf{E X} f^{*}$, and $q_{1}{ }^{H M L_{n+1}} q_{2}$ implies $q_{2} \vDash \mathbf{E X} f^{*}$, which means that there exists some $q_{2} \rightarrow q_{2}^{\prime}$ with $q_{2}^{\prime} \models f^{*}$. Now, if both $q_{1}^{\prime}$ and $q_{2}^{\prime}$ satisfy $f^{*}$, we clearly have $q_{1}^{\prime H M L_{m}} q_{2}^{\prime}$ by construction of $f^{*}$, which implies $q_{1}^{\prime} \equiv_{n} q_{2}^{\prime}$ (by ind. hyp.). This establishes that $q_{1} \equiv_{n+1} q_{2}$. Essentially the same proof appears in [Mil81,GR83].

$(\Leftarrow)$ : Clearly $\equiv_{0} \subseteq \stackrel{H M L_{0}}{\sim}$, so assume $\equiv_{n} \subseteq{ }^{H M L_{n}}$. Consider $q_{1} \equiv_{n+1} q_{2}$ and $q_{1} \vDash f$. We show that $q_{2}=f$ by induction on the structure of $f \in H M L_{n+1}$. This is obvious if $f$ has the form $\mathrm{T}, g \wedge g^{\prime}$ or $\neg g$. If $f$ is $\mathbf{E X} g$ with $g \in H M L_{n}$ then $q_{1} \models f$ implies that there exists $q_{1} \rightarrow q_{1}^{\prime}$ with $q_{1}^{\prime} \neq g$. Now, $q_{1} \equiv_{n+1} q_{2}$ implies that there exists $q_{2} \rightarrow q_{2}^{\prime}$ with $q_{1}^{\prime} \equiv_{n} q_{2}^{\prime}$, that is $q_{1}^{\prime z M L_{n}} q_{2}^{\prime}$. Then $q_{2}^{\prime} \vDash g$ and $q_{2} \vDash \mathbf{E X} g$, that is $q_{2} \vDash f$. Finally, $q_{1}{ }^{H M L_{n+1}} q_{2}$.

This lemma entails that $\stackrel{H M L}{\sim}=\equiv$ and has the immediate corollary:

Proposition 2 For any graph $(Q, \rightarrow)$, and states $q_{1}, q_{2} \in Q$ :

$$
q_{1} \stackrel{C T L}{\sim} q_{2} \Rightarrow q_{1} \equiv q_{2}
$$

Of course, the reverse implication does not hold, as a corollary of Proposition 4.

Remark 1 [HM85] proves ${ }^{H M L} \sim=\equiv$ for labeled f.b. graphs, while we proved it for unlabeled non-f.b. graphs. Oddly enough, the " $\Rightarrow$ " direction does not hold for labeled non-f.b. graphs when the labeling alphabet is infinite (see Appendix B).

\section{Comparing $C T L$ and $\approx$}

We first exhibit a graph where two states are $\stackrel{\operatorname{crL}}{\sim}$ without being $\approx$. We consider the ordinal process $P_{\lambda}$ with $\lambda$ infinite and first establish the following lemma:

Lemma 2 For any $f \in C T L$ and any $\alpha \geq d(f)$ in $P_{\lambda}, \alpha \models f$ iff $d(f) \models f$

In other words, in $P_{\lambda}$ any $f \in C T L$ is always true (or always false) from the integer state $d(f)$ onwards.

Proof We prove the lemma by induction over the structure of $f$. 
- Assume $f=\mathbf{E X} g$ and $n=d(g)$, and consider $\alpha \geq n+1$. If $n+1 \models f$, then $\alpha \models f$. If $n+1 \not \neq f$ then $k \not \neq g$ for any $k \leq n$, which implies $n \not \neq g$ and then, by ind. hyp., $\beta \not \neq g$ for any $\beta \geq n$ because $n=d(g)$. Finally, $\alpha \not \neq f$.

- Assume $f=\mathbf{E} g_{1} \mathbf{U} g_{2}$ and $n=d(f)$, and consider $\alpha \geq n$. First suppose that $n \models f$. Then $n \models g_{1}$ or $n \vDash g_{2}$. If $n \vDash g_{2}$ then $\alpha \models g_{2}$ (by ind. hyp.) and then $\alpha \models f$. Otherwise $n \models g_{1}$, and there exists $n \rightarrow k$ with $k \models g_{2}$. Now $\alpha \models g_{1}$ (by ind. hyp.) and as $\alpha \rightarrow k$, we have $\alpha \models f$. The other possibility is $n \not \models f$, and then $n \not g_{2}$, implying $\alpha \not g_{2}$ (by ind. hyp.). If now $\alpha \models f$ then $\alpha \models g_{1}$ (and then $n \vDash g_{1}$ ) and there exists $\alpha \rightarrow \beta$ with $\beta \models g_{2}$. As $\beta$ must be less than $n$ or else we would have $n \vDash g_{2}$, we have $n \rightarrow \beta$, contradicting $n \not \neq f$.

A similar proof applies to the $\mathbf{A} g_{1} \mathbf{U} g_{2}$ case, and the remaining cases are obvious.

This lemma has the immediate corollary:

Proposition $3 q_{1}{ }^{C T L} q_{2}$ does not imply $q_{1} \approx q_{2}$

Indeed, the previous lemma shows that in $P_{\omega+2}, \omega_{\sim}^{c \tau t} \omega+1$, while $\omega \neq \omega \omega+1$.

We now consider the graph $P$ obtained from the ordinal process $P_{\omega^{2}+2}$ by adding a transition $\omega^{2}+1 \rightarrow \omega^{2}+1$. We begin with the following lemma:

Lemma 3 For all $n \in \mathrm{N}$ and $\alpha_{1}, \alpha_{2} \geq \omega . n$, we have $\alpha_{1} \approx_{n} \alpha_{2}$ in $P$.

Proof We proceed by induction over $n$. The result is clear for $n=0$, so assume it holds for $n$ and consider $\alpha_{1}, \alpha_{2} \geq \omega .(n+1)$. If $\alpha_{1} \stackrel{k}{\rightarrow} \beta_{1}, \beta_{1}$ may be less than $\omega . n$ or not. If $\beta_{1}<\omega . n$, then also $\alpha_{2} \stackrel{k}{\rightarrow} \beta_{1}$. If $\beta_{1} \geq \omega . n$ then we can consider $\alpha_{2} \stackrel{k}{\rightarrow} \omega . n$ and have $\beta_{1} \approx_{n} \omega . n$ by ind. hyp. Finally we obtain $\alpha_{1} \approx_{n+1} \alpha_{2}$, which completes the proof.

The same proof generalizes the result we mentioned after Definition 4 by allowing any transitions $\alpha_{1} \rightarrow \alpha_{2}$ to be added in $P_{\lambda}$, provided that $\alpha_{1}$ and $\alpha_{2}$ are above $\omega^{2}$. This lemma has the immediate corollary:

Proposition $4 q_{1} \approx q_{2}$ does not imply $q_{1} \stackrel{\text { cTL }}{\sim} q_{2}$

Indeed, we just saw that in $P, \omega^{2} \approx \omega^{2}+1$, though there exists an infinite path starting from $\omega^{2}+1$ and none from $\omega^{2}$. This is easily expressed in $C T L$ and for $f=\mathbf{A} T \mathbf{U} \neg \mathbf{E X} \top$ we have $\omega^{2} \vDash f$ and $\omega^{2}+1 \not \neq f$.

\section{Conclusion}

As stated in the introduction, this paper was written to answer some questions that arose naturally in the semantics of FP2 [SJ89]. The implications of our findings are clear: we cannot use $C T L$ to reason about FP2 programs without adopting a semantics at least as discriminating 
as $\stackrel{C T L}{\sim}$ (e.g. bisimulation). The use of $\approx$ or $\equiv$ (as in [GR83,BBK87]) is simply not compatible with $C T L$. Furthermore we cannot use $\stackrel{c T L}{\sim}$ itself as a semantic equivalence for it is not a congruence [Sch89].

Further, we think these results are more widely interesting. They illustrate the strength of the f.b. restriction. Indeed, several examples suggest that the equivalences generated by temporal logics are very sensitive to this restriction [BR83,Sch89].

The ordinal processes were used by Klop to show that $\approx$ and $\leftrightarrow$ do not coincide: the earlier proof of [San82] used much more complicated counter-examples. In this paper they provided us with "simple" generic counter-examples. Indeed, the study of process theory and process logics in the non-f.b. framework requires such a collection of paradigmatic examples and counterexamples. We believe that the temporal theory of ordinal graphs deserves to be studied in its own right, generalizing results like Lemmas 2 and 4 .

\section{Acknowledgements}

We would like to thank Hubert Comon for his patient and thorough criticism of the draft.

\section{Appendix}

\section{A The $C T L^{*} \operatorname{logic}$}

In this section, we show that the results we developed for $C T L$ are still valid for $C T L^{*}$, a richer logic introduced in [EH83]. In the paper, we preferred to concentrate on CTL because it has a very simple presentation, and does not mix state and path formulas the way $C T L^{*}$ does. Indeed, the use of path formulas in $C T L^{*}$ leads one to define in a less direct way the equivalence generated over states.

We follow Stirling and define $C T L^{*}$ in the following way:

\section{Definition 7 (Syntax of $C T L^{*}$ )}

$$
\left(C T L^{*} \ni\right) f, g::=T|f \wedge g| \neg f|\mathbf{X} f| f \mathbf{U} g \mid \forall f
$$

where now truth is defined w.r.t. to a path $\sigma$ of $(Q, \rightarrow)$.

\section{Definition 8 (Semantics of $C T L^{*}$ )}

- $\sigma \models T, \sigma \models f \wedge g$ and $\sigma \models \neg f$ have their obvious definitions.

- $\sigma \vDash \mathbf{X} f$ iff $|\sigma| \geq 1$ and $\sigma^{1} \vDash f$.

- $\sigma \models f \mathbf{U} g$ iff there exists $n \in \mathbf{N}$ s.t. $|\sigma| \geq n, \sigma^{n} \models g$ and $\sigma^{i} \vDash f$ for all $0 \leq i<n$.

- $\sigma \models \forall f$ iff $\sigma^{\prime} \models f$ for all $\sigma^{\prime} \in \Sigma(\sigma(0))$. 
Given $q \in Q$, we write $q \models f$ iff $\sigma \models f$ for all $\sigma \in \Sigma(q)$. Note that $q \not \models f$ does not imply $q \models \neg f$. Again, we write $\sigma^{c r L^{*}} \sim \sigma^{\prime}$ and $q^{c \tau L^{*}} \sim q^{\prime}$ with obvious definitions.

$C T L^{*}$ subsumes $C T L$ in the sense that every formula $f \in C T L$ can be directly translated into a formula $f^{*} \in C T L^{*}$ s.t. $q \models_{C T L} f$ iff $q \models_{C T L *} f^{*}$. This is easy and well-known.

The modal height $d(f)$ of $C T L^{*}$ formulas is defined as with $C T L: d(\forall f)=d(f)$ and $d(f \cup g)=\max (d(f), d(g))$.

Proposition 5 For any graph $(Q, \rightarrow)$, and paths $\sigma_{1}, \sigma_{2} \in \Sigma$ :

$$
\sigma_{1} \leftrightarrows \sigma_{2} \Rightarrow \sigma_{1} \stackrel{C T L^{*}}{\sim} \sigma_{2}
$$

Again, the proof is by induction over the structure of a formula $f \in C T L^{*}$. This proposition has the immediate corollary:

Proposition 6 For any graph $(Q, \rightarrow)$, and states $q, q^{\prime} \in Q$ :

$$
q \biguplus q^{\prime} \Rightarrow q^{C T L^{*}} q^{\prime}
$$

The reverse implication does not hold in general: a simple cardinality argument shows that $C T L^{*}$ cannot distinguish between more than $\aleph_{1}$ states while there exist ordinal graphs of larger size. However, we believe the stronger:

Conjecture 1 In $P_{\lambda}, \omega^{C T L^{*}} \omega+1$.

which would entail

$$
q_{1} \stackrel{c T L^{*}}{\sim} q_{2} \text { does not imply } q_{1} \approx q_{2}
$$

We uncovered an error in our earlier proof of Conjecture 1. In particular, an extension to $C T L^{*}$ of Lemma 2 proved incorrect:

Lemma 4 There exist formulas $f \in C T L^{*}$ and states $\alpha \geq d(f)$ in $P_{\lambda}$ such that $\alpha \not f$ while $d(f) \models f$

We need some notation for our example. Write $(\geq n)$ for $\neg \forall \neg X^{n} T,(=n)$ for $(\geq n) \wedge \neg(\geq n+1)$ and $(\ni n)$ for $\mathrm{TU}(=n)$. Then $\sigma \models(\geq n)$ iff $\sigma(0) \geq n, \sigma \models(=n)$ iff $\sigma(0)=n$ and $\sigma \models(\ni n)$ iff $\sigma(i)=n$ for some $i \leq|\sigma|$. Now consider

$$
f_{n}=(\ni n-1) \vee \mathbf{X} \neg(\geq n-1)
$$

$f_{n}$ has depth $n$. For $n \geq 2$ in $P_{\lambda}$, we have

$$
n \models f \text { while } n+1 \not f
$$

There exist other examples where the strict $n=d(f)$ is not required. Consider

$$
f_{n}=\neg \mathbf{X X} \top \vee(\ni n-2) \vee(\ni n-3) \vee \mathbf{X X} \neg(\geq n-3)
$$

where $d\left(f_{n}\right)=n-1$ and where (6) holds for $n \geq 3$. 


\section{B Labeled graphs}

We assume an infinite set $A=\{a, \ldots\}$ of action names. A graph is now a pair $(Q, \rightarrow)$ where $\rightarrow \subseteq Q \times A \times Q$. We write $q \stackrel{a}{\rightarrow} q^{\prime}$ when $\left(q, a, q^{\prime}\right) \in \rightarrow$. The definitions of bisimulation and of the two other equivalences are adapted in the usual way. The first problem is to incorporate these actions in the temporal logic. The Hennessy-Milner Logic has one modal combinator per action: EX is replaced by all $\langle a\rangle$ (for $a \in A$ ), with the semantics:

$$
q \models\langle a\rangle f \text { iff } \exists q \stackrel{a}{\rightarrow} q^{\prime} \text { s.t. } q^{\prime} \vDash f
$$

However, this only allows references to actions in the EX combinator, not in the two other ones. A more general solution is to include atomic propositions "after $a$ " (for any $a \in A$ ) with the semantics:

$$
q \models \text { after } a \text { iff } \exists q^{\prime} \stackrel{a}{\rightarrow} q
$$

Note that $\langle a\rangle f$ can be written EX $(f \wedge$ after $a)$. We adopt this extension and end up with a new logic: $C T L_{A}$. As we expect the semantics of after $a$ to match our intuition, we restrict ourselves to labeled graphs that are trees. A rooted-graph can be unfolded into an equivalent (bisimilar) tree, so that there is no loss of generality.

Of course all our negative results still hold in this framework. Furthermore, we still have:

$$
q_{1} \leftrightarrows q_{2} \Rightarrow q_{1} \stackrel{C r L}{\sim} q_{2}
$$

with an almost identical proof.

More interesting is the following:

$$
q_{1} \stackrel{H M L \wedge}{\sim} q_{2} \underset{q_{1}}{\rightleftharpoons} q_{2}
$$

where the " $\Leftarrow$ " direction is proved exactly as in Lemma 1 . The " $\Rightarrow$ " direction only holds when $A$ is finite. Our counter-example needs some more definitions. We write $\mathcal{P}(A)$ for the sets of subsets of $A$ and $\mathcal{P}_{c f}(A)$ for the sets of finite or cofinite ${ }^{1}$ subsets of $A$. Given $B \subseteq A$, we define $P_{B}=\Sigma_{b \in B} b . N i l$ (with standard CCS-like definitions for + , action-prefixing and $N i l$ ). Note that $P_{B} \equiv_{1} P_{B^{\prime}}$ iff $B=B^{\prime}$. Now, if we pick any $a \in A$ and define $P=\Sigma_{B \in \mathcal{P}(A)} a . P_{B}$ and $P^{\prime}=\Sigma_{B \in \mathcal{P}_{c f}(A)} a . P_{B}$, we have $P^{H M L_{A}} P^{\prime}$ while $P \not_{2} P^{\prime}$. (We do not include the proof).

As we also have $P^{C T L} \sim P^{\prime}$, we end up with:

$$
q_{1} \stackrel{\operatorname{Crt}_{A}}{\sim} q_{2} \nRightarrow q_{1} \equiv q_{2}
$$

\section{References}

[Abr87] S. Abramsky. Observation equivalence as a testing equivalence. Theoretical Computer Science, 53:225241, 1987.

[BBK87] J. C. M. Baeten, J. A. Bergstra, and J. W. Klop. On the consistency of Koomen's fair abstraction rule. Theoretical Computer Science, 51(1):129-176, 1987.

\footnotetext{
${ }^{1}$ i.e. having a finite complement.
} 
[BK87] J. A. Bergstra and J. W. Klop. A Convergence Theorem in Process Algebra. Research Report CSR8733, CWI, 1987.

[BK89] J. A. Bergstra and J. W. Klop. Process theory based on bisimulation semantics. In Linear Time, Branching Time and Partial Order in Logics and Models for Concurrency, Noordwijkerhout, LNCS \$54, pages 50-122, Springer-Verlag, 1989.

[BR83] S. D. Brookes and W. C. Rounds. Behavioural equivalence relations induced by programming logics. In Proc. 10th ICALP, Barcelona, LNCS 154, pages 97-108, Springer-Verlag, July 1983.

[CE81] E. M. Clarke and E. A. Emerson. Design and synthesis of synchronization skeletons using branching time temporal logic. In Proc. Logics of Programs Workshop, Yorktown Heights, LNCS 191, pages 5271, Springer-Verlag, May 1981.

[EH83] E. A. Emerson and J. Y. Halpern. "Sometimes" and "Not Never" revisited: on branching versus linear time. In Proc. 10th ACM Symp. Principles of Programming Languages, Austin, Texas, pages 127-140, January 1983.

[ES89] E. A. Emerson and J. Srinivasan. Branching time temporal logic. In Linear Time, Branching Time and Partial Order in Logics and Models for Concurrency, Noordwijkerhout, LNCS 354, pages 123-172, Springer-Verlag, 1989.

[GR83] W. G. Golson and W. C. Rounds. Connections between two theories of concurrency: metric spaces and synchronization trees. Information and Control, 57, 1983.

[HM85] M. Hennessy and R. Milner. Algebraic laws for nondeterminism and concurrency. Journal of the ACM, 32(1):137-161, January 1985.

[HS85] M. Hennessy and C. Stirling. The power of the future perfect in program logics. Information and Contral, 67:23-52, 1985.

[Klo88] J. W. Klop. Bisimulation Semantics. Lectures given at the REX School/Workshop, Noordwijkerhout, NL, May 1988.

[Lam80] L. Lamport. "Sometimes" is sometimes "Not Never". In Proc. 7th ACM Symp. Principles of Programming Languages, Las Vegas, pages 174-185, January 1980.

[Mil81] R. Milner. A modal characterisation of observable machine-behaviour. In Proc. CAAP 81, Genoa, LNCS 185, pages 25-34, Springer-Verlag, March 1981.

[Mil88] R. Milner. Operational and Algebraic Semantics of Concurrent Processes. Research Report ECSLFCS-88-46, Lab. for Foundations of Computer Science, Edinburgh, February 1988. To appear in the Handbook of Theoretical Computer Science.

[Par81] D. Park. Concurrency and automata on infinite sequences. In Proc. 5th GI Conf., pages 167-183, Springer-Verlag, March 1981.

[Pnu77] A. Pnueli. The temporal logic of programs. In Proc. 18th IEEE Symp. Foundations of Computer Science, Providence, pages 46-57, 1977.

[San82] M. T. Sanderson. Proof Techniques for CCS. PhD thesis, Univ. Edinburgh, November 1982. Available as Report CST-19-82.

[Sch89] Ph. Schnoebelen. Congruence Properties of the Process Equivalence Induced by Temporal Logic. 1989. Submitted for publication.

[SJ89] Ph. Schnoebelen and Ph. Jorrand. Principles of FP2. Term algebras for specification of parallel machines. In J. W. de Bakker, editor, Languages for Parallel Architectures: Design, Semantics, Implementation Models, chapter 5, pages 223-273, Wiley, 1989. 